Quim. Nova, Vol. 33, No. 3, 716-720, 2010

\title{
MARCADORES METÁLICOS COMO AVALIAÇÃO DO IMPACTO CRÔNICO DE EMISSÕES PETROQUÍMICAS EM ZONA URBANA
}

\author{
Felipe Azevedo de Paula Garcia*, Nicolai Mirlean e Paulo Roberto Baisch \\ Instituto Oceanográfico, Universidade Federal do Rio Grande, Av. Itália, km 08, s/n, 96201-900 Rio Grande - RS, Brasil
}

Recebido em 4/1/09; aceito em 19/8/09; publicado na web em 12/1/10

\begin{abstract}
METALLIC TRACERS AS AN EVALUATION TOOL FOR LONG-TERM PETROCHEMICAL EMISSIONS IN URBAN ENVIRONMENT. Nickel and vanadium were tested as tracers of oil refinery emission distribution in populated area of Rio GrandeRS. The anomalies of these elements in surface soil are considered a long-term reflection of the emissions in low atmosphere. The spatial distribution of $\mathrm{Ni}$ and $\mathrm{V}$ in the soil corresponded to the pattern of local winds. The threshold of 1.5 backgrounds of these metals markedly outlined the area of petrochemical emissions. Anomalies of $\mathrm{Ni}$ and $\mathrm{V}$ in surface soil have a rather different configuration in comparison with other metal tracers of urban impact: $\mathrm{Cd}, \mathrm{Cu}, \mathrm{Pb}$ and $\mathrm{Zn}$.
\end{abstract}

Keywords: metal markers; petro-chemistry emission; urban environment.

\section{INTRODUÇÃO}

O monitoramento e a avaliação do impacto das emissões da indústria petrolífera são importantes devido ao efeito negativo sobre o meio ambiente e população. ${ }^{1-5}$ Considerando-se a proximidade entre áreas residenciais e industriais, os efeitos nocivos dos elementos e compostos oriundos de emissões atmosféricas têm consequências sobre a saúde pública e a qualidade ambiental. A exposição de organismos a compostos aromáticos presentes no petróleo pode gerar efeitos carcinogênicos e mutagênicos. ${ }^{1}$ Ademais, a exposição de organismos às cinzas geradas pela combustão de óleo pode acarretar lesões pulmonares. ${ }^{2}$

Entre os métodos de monitoramento e controle da dispersão de emissões industriais mais frequentemente usados destacam-se: modelagem numérica; monitoramentos da qualidade do ar e precipitações através de coleta periódica de amostras por meio de vários tipos de coletores. ${ }^{6-9}$

Quanto à modelagem numérica de dispersão, verifica-se uma lenta, mas contínua melhora na capacidade de previsão meteorológica. Essa melhora é devida não só à compreensão de processos importantes no campo da meteorologia, mas também ao rápido aumento da capacidade computacional. No entanto, a elaboração de modelos e sua validação encontram problemas significativos em áreas urbanas, onde a topografia irregular (devido à diversidade de edificações) modifica significativamente as componentes do movimento das correntes de ar junto ao solo, entorno de $1,5 \mathrm{~m}$, na altura da respiração do homem. Modelos aplicados a regiões urbanas possuem um erro na estimativa de duas a três vezes as medições com a rede de coletores. ${ }^{10}$ Também se verifica que os modelos numéricos de dispersão em áreas edificadas, ao nível do solo, podem tanto superestimar as concentrações como subestimar o alcance dos contaminantes. ${ }^{11}$

Da mesma maneira, a necessidade das amostragens pontuais para diagnóstico e monitoramento das emissões em geral requer o emprego de equipamentos com alto custo de aquisição e operação. Na cidade de Rio Grande atualmente existem apenas três estações de amostragem de material particulado atmosférico ( $\mathrm{Hi}$ vol - FEPAM) para tentar abranger toda a área urbana e industrial. Este quadro determina a inviabilidade do uso de malhas amostrais de grande densidade e, assim, torna-se necessário um planejamento estratégico específico.

\footnotetext{
*e-mail: felipeoceanofurg@ gmail.com
}

Uma alternativa para modelagem e monitoramento por meio de coletores, especialmente para detecção de zonas sujeitas à exposição de longo prazo, é o emprego de segmentos do sistema ambiental para análise de marcadores específicos e persistentes. O uso de elementos metálicos como marcadores persistentes permite a identificação de fontes de contaminação atmosférica de amplitude continental, ${ }^{12}$ mas no caso de processos locais, especialmente para a indústria petroquímica, são utilizados mais comumente os marcadores orgânicos..$^{13}$ Os marcadores inorgânicos, no entanto, têm a vantagem de não serem degradáveis, sendo mais persistentes e, consequentemente, mais eficientes na avaliação de impactos crônicos.

Os elementos metálicos níquel e vanádio são conhecidos por serem integrantes naturais do petróleo, assim seus teores e a abundância relativa são indicativos da qualidade e maturação do petróleo. ${ }^{14}$ Da mesma maneira são indicadores do impacto ambiental causado por derrames ${ }^{3,4}$ e combustão $0^{4,8,9,15}$ de produtos petroquímicos e seus derivados.

Apesar desse estudo utilizá-los essencialmente como marcadores ambientais, deve-se destacar que Ni e V demonstram efeitos tóxicos e sinérgicos importantes sobre a saúde, ocasionando alterações fisiológicas, tais como braquicardia e hipotermia. ${ }^{5}$

No entanto, não há no Brasil registro de uso desses marcadores inorgânicos para avaliação do impacto crônico das atividades petroquímicas.

A indústria petroquímica no Brasil vem se desenvolvendo especialmente em zonas costeiras, devido às facilidades portuárias e à extração do petróleo no mar. Essas áreas são as que apresentam, no mundo, as maiores densidades demográficas, acarretando, em especial no Brasil, uma sobreposição de atividades de natureza residencial e industrial petroquímica. Em contrapartida, os instrumentos legais de controle, como as licenças de operação dos empreendimentos petroquímicos, por vezes são pouco restritivos. No caso da refinaria estudada, a $\mathrm{LO}^{16}$ versa somente sobre procedimentos, como aplicação de vapor para minimizar as emissões, e restrições quanto à frequência e duração das emissões visíveis.

A cidade do Rio Grande (RS) apresenta uma grande densidade populacional nas proximidades de uma empresa de refino de petróleo que existe há mais de 70 anos, o que destaca a importância da poluição atmosférica oriunda dessa indústria. Desta forma, o município de Rio Grande apresenta um quadro ambiental muito adequado para um estudo utilizando marcadores persistentes em área urbana. Ademais, o diagnóstico de impactos crônicos pode servir de subsídio 
para investigações mais detalhadas em termos de sazonalidade e da ocorrência de contaminantes orgânicos.

O presente trabalho teve como foco investigar a possibilidade do uso da distribuição do Ni e V em solos superficiais urbanos como marcadores das zonas de impacto crônico causadas pelas emissões petroquímicas na atmosfera, em uma região densamente urbanizada.

\section{PARTE EXPERIMENTAL}

\section{Área de estudo}

O município de Rio Grande está localizado na zona estuarina da Lagoa dos Patos (RS) próximo à desembocadura no Oceano Atlântico e possui uma população de cerca de 190 mil habitantes, com taxa de urbanização superior a $95 \% .{ }^{17}$

A refinaria de petróleo considerada nesse estudo é a mais antiga do Brasil. Foi construída em meados de década de 1930, e teve suas instalações progressivamente envolvidas por áreas residenciais, resultando na atual configuração de zoneamento urbano (Figura 1S, material suplementar). As fontes de emissão da refinaria mudaram pouco ao longo de sua história, podendo ser consideradas fixas durante as últimas dezenas de anos.

Os ventos predominantes na região são de nordeste durante o verão, e da direção sul durante o inverno, contudo, os ventos de nordeste são os dominantes ao longo do ano.

No que tange à geomorfologia, a região é formada por terraços lagunares, campos de dunas e barreiras lagunares. Especificamente na área urbana, foco desse estudo, essas características estão ausentes pela ocorrência de aterros, portanto, não existe a formação de solo propriamente dito. A textura do material no solo urbano é arenosa, rica em silicatos. Na região entorno encontram-se solos podzolicos ou areia quartzosa, sendo suas características geoquímicas as mais adequadas para eventuais comparações.

\section{Amostragem}

Foram coletadas, entre os meses de abril e maio de 2008, um total de 98 amostras de solo nos arredores da refinaria, incluindo áreas urbana e industrial, em um raio máximo de 1,3 km das fontes de emissão (Figura 1S, material suplementar).

Para a coleta das amostras foram selecionados locais não alterados por aterros recentes, trânsito de pessoas, animais e/ou veículos e demais atividades que pudessem interferir na qualidade da amostra. Foram coletados os primeiros centímetros da camada superficial do solo $(0-5 \mathrm{~cm})$ com o auxílio de uma pá de plástico. Essa camada foi escolhida, pois os marcadores investigados têm baixa tendência de serem lixiviados, acumulando-se na camada superficial dos solos. ${ }^{4}$

As amostras foram acondicionadas em sacos plásticos e transportadas ao laboratório, onde foram secas em estufas à temperatura de $80^{\circ} \mathrm{C}$. Após a secagem, as amostras foram desagregadas e peneiradas manualmente em malha de nylon de $63 \mu \mathrm{m}$.

Tendo em vista que os solos da região são essencialmente quartzoarenosos procedeu-se à peneiragem com o objetivo de remover o ma- terial inerte, que tende a diminuir a concentração dos contaminantes e, assim, dificultar a interpretação ambiental. Ademais, as partículas mais finas são as que carreiam a maior parte dos contaminantes, assim como possuem maior capacidade de serem ressuspensas, podendo ser vetores de contaminação através da respiração humana.

\section{Análises químicas}

As amostras de solo (fração < $63 \mu \mathrm{m}$ ) foram submetidas à digestão química, com sucessivos ataques a quente de $\mathrm{HNO}_{3}, \mathrm{H}_{2} \mathrm{O}_{2}$ e $\mathrm{HCl}$, conforme o método EPA 3050b. ${ }^{18}$

$\mathrm{Ni}, \mathrm{Cu}, \mathrm{Pb}$ e $\mathrm{Zn}$ foram determinados por espectrofotometria de absorção atômica por chama ar-acetileno, utilizando-se um aparelho GBC 932AA, enquanto que $\mathrm{V}$ e $\mathrm{Cd}$ foram determinados por espectrofotometria de absorção atômica através de atomização eletrotérmica (GF3000). A precisão e exatidão da análise foram aprovadas pela análise paralela de amostra de referência MESS- ${ }^{19}$ (Tabela 1). A análise demonstrou boa recuperação dos valores de referência na faixa de $95 \%$. Todas as medidas foram feitas com controle de reprodutividade entre as réplicas com desvio padrão menor que $5 \%$.

\section{Nível de referência e coeficientes de enriquecimento}

Dentre as diversas técnicas para a determinação de valores de background e níveis de referência, ${ }^{20}$ utilizou-se o valor de concentração correspondente ao primeiro pico modal da distribuição como background urbano (BGurb) dos elementos metálicos dos solos.

$\mathrm{O}$ background urbano não tem o objetivo de estabelecer os níveis naturais dos elementos metálicos estudados na região. Esse valor corresponde ao nível de impactação pelos elementos estudados resultante das diversas fontes urbanas. Desta forma, o impacto por metais da indústria de refino de petróleo ficará sobreposto ao BGurb, podendo assim ser identificado.

Os coeficientes de enriquecimento (Kurb) foram determinados a partir da razão do teor da amostra pelo valor do BGurb.

\section{Dados meteorológicos}

Para comparação dos padrões de distribuição dos elementos com os fenômenos meteorológicos foi utilizada uma série de dados coletados pela Estação Meteorológica n ${ }^{\circ} 83995$, de Rio Grande, operada pelo Departamento de Geociências da FURG em convênio com o $8^{\circ}$ Distrito de Meteorologia do Instituto Nacional de Meteorologia.

A série de dados utilizada representa três medidas diárias de velocidade e direção dos ventos entre 01/01/1990 e 31/12/2007.

\section{Tratamento estatístico}

A similaridade da presença dos elementos na área de estudo foi testada utilizando uma análise de Cluster, usando software ${ }^{21}$ complementar ao MS Excel. ${ }^{22}$

Com os resultados dos Kurb foram gerados mapas de distribuição, utilizando software para interpolação (método Kriging) de resultados e produção de gráficos. ${ }^{23}$

Tabela 1. Valores certificados para o material de referência ${ }^{19} \mathrm{e}$ a taxa recuperação nos ensaios analíticos

\begin{tabular}{|c|c|c|c|c|c|c|}
\hline MESS-3 & $\mathbf{N i}$ & $\mathbf{V}$ & $\mathbf{P b}$ & Cd & Zn & $\mathbf{C u}$ \\
\hline Concentração Certificada & $46,9 \mathrm{mg} / \mathrm{kg}$ & $243 \mathrm{mg} / \mathrm{kg}$ & $21,1 \mathrm{mg} / \mathrm{kg}$ & $0,24 \mathrm{mg} / \mathrm{kg}$ & $159 \mathrm{mg} / \mathrm{kg}$ & $33,9 \mathrm{mg} / \mathrm{kg}$ \\
\hline IC $95 \%$ & 2,2 & 10 & 0,7 & 0,01 & 8 & 1,6 \\
\hline Recuperação & $101 \%$ & $98 \%$ & $97 \%$ & $102 \%$ & $99 \%$ & $99 \%$ \\
\hline $\mathrm{N}(\mathrm{RSD})$ & $12(4,3 \%)$ & $12(0,9 \%)$ & $12(2,9 \%)$ & $12(0,7 \%)$ & $12(4,8 \%)$ & $12(3,9 \%)$ \\
\hline
\end{tabular}

IC, intervalo de confiança; $\mathrm{N}$, número de réplicas; $\mathrm{RSD}$, desvio padrão entre as réplicas 


\section{RESULTADOS E DISCUSSÃO}

A análise dos 6 metais nos pontos amostrados apresentou concentrações com distribuições distintas. As médias, desvio padrão e alcance dos teores encontrados no estudo para cada metal estão descritos na Tabela 2.

Tabela 2. Média, desvio padrão, máximos e mínimos dos teores metálicos identificados, valores de $\mathrm{BG}_{\text {urb }}$ calculados, $\mathrm{BG}$ natural para a região identificado por Vanz ${ }^{25}$ e BG para solos podzolicos relatados na ref. 24 (todos em mg/kg)

\begin{tabular}{lccccc}
\hline & Média (DP) & Alcance & BG $_{\text {urb }}$ & $\begin{array}{c}\text { BG } \\
\text { Vanz }^{25}\end{array}$ & $\begin{array}{c}\text { BG Beus } \\
\text { et al. } .^{24}\end{array}$ \\
\hline $\mathrm{Ni}$ & $31,25(16,74)$ & $10,33-112,38$ & 25 & 7,4 & 30 \\
$\mathrm{~V}$ & $48,45(23,30)$ & $19,10-159,40$ & 40 & 35 & 63 \\
$\mathrm{Zn}$ & $514,15(242,01)$ & $102,20-1614,42$ & 400 & 21,9 & 41 \\
$\mathrm{Cu}$ & $124,71(118,32)$ & $24,94-826,75$ & 100 & 5,8 & 15 \\
$\mathrm{~Pb}$ & $234,97(185,02)$ & $38,11-918,90$ & 120 & 2,7 & 11 \\
$\mathrm{Cd}$ & $0,96(0,76)$ & $0,16-0,52$ & 0,45 & $\mathrm{xx}$ & 0,50 \\
\hline
\end{tabular}

Para a determinação do BGurb foi utilizada a distribuição das ocorrências dos teores conforme classes de teor (de acordo com suas distribuições em diferentes classes). Definiu-se como o valor de BGurb o primeiro pico de ocorrências, estabelecendo-se os valores de $0,45,25$, 40, 100, 120 e $400 \mathrm{mg} \mathrm{kg}^{-1}$ para Cd, Ni, V, Cu, Pb e Zn, respectivamente (Figura 2S, material suplementar). Segundo Beus et al.,${ }^{24} \mathrm{o}$ background mundial para $\mathrm{Cd}, \mathrm{Ni}, \mathrm{V}, \mathrm{Cu}, \mathrm{Pb}$ e $\mathrm{Zn}$ em solos arenosos do tipo podsol (Tabela 2) são, respectivamente, 0,50; 30; 63; 15; 11 e $41 \mathrm{mg} \mathrm{kg}^{-1}$.

A comparação destes valores indica que os solos urbanos na área estudada não estão, em geral, contaminados por $\mathrm{Ni}, \mathrm{V}$, e Cd. Mas a contaminação por $\mathrm{Zn}, \mathrm{Cu}$ e $\mathrm{Pb}$ é considerada significativa e abrange a totalidade da região urbana estudada.

Na Tabela 2 estão também incluídos os valores do background dos metais pesados para a região, calculados a partir de solos arenosos não contaminados. ${ }^{25}$ Verifica-se que os valores encontrados nesse trabalho são muito superiores, indicando que o impacto da urbanização é significativo para todos os elementos.

A análise de cluster foi testada com a similaridade conforme a correlação de Pearson (Tabela 3), demonstrando uma forte relação direta entre os elementos $\mathrm{Ni} \mathrm{e} \mathrm{V}(\mathrm{r}=0,88$; $\mathrm{p}<0,05, \mathrm{n}=98)$ e uma relação nula ou inversa desses elementos com os demais metais. A alta correlação ente Ni e V na área evidencia uma fonte comum para os dois metais. Além disso, os valores de BGurb calculados assemelham-se aos valores de BG mundial para ambos os elementos, indicando a ausência de demais fontes desses elemento na região. Os outros metais, como $\mathrm{Pb}$ e $\mathrm{Zn}$, que não apresentaram correlação significativa com o cluster do Ni e V, também demonstraram correlação significativa e positiva entre $\mathrm{si}(\mathrm{r}=0,71 ; \mathrm{p}<0,05 ; \mathrm{n}=98)$. A correlação positiva entre $\mathrm{Pb}$ e $\mathrm{Zn}$ em solos contaminados e em precipitações sólidas urbanas é bem conhecida, sendo o resultado da paragênese destes elementos em áreas urbanas. ${ }^{26}$

A Figura 1 apresenta a distribuição dos elementos estudados na camada superficial de solo. O Zn (Figura 1F) apresentou uma

Tabela 3. Matriz de similaridade conforme correlação de Pearson

\begin{tabular}{lcccccc}
\hline & $\mathrm{Ni}$ & $\mathrm{V}$ & $\mathrm{Zn}$ & $\mathrm{Cu}$ & $\mathrm{Pb}$ & $\mathrm{Cd}$ \\
\hline $\mathrm{Ni}$ & $\mathrm{X}$ & 0.876 & 0.125 & 0.098 & 0.000 & -0.007 \\
$\mathrm{~V}$ & 0.876 & $\mathrm{X}$ & 0.006 & 0.090 & -0.058 & -0.006 \\
$\mathrm{Zn}$ & 0.125 & 0.006 & $\mathrm{X}$ & 0.202 & 0.705 & 0.338 \\
$\mathrm{Cu}$ & 0.098 & 0.090 & 0.202 & $\mathrm{X}$ & 0.221 & 0.209 \\
$\mathrm{~Pb}$ & 0.000 & -0.058 & 0.705 & 0.221 & $\mathrm{X}$ & 0.163 \\
$\mathrm{Cd}$ & -0.007 & -0.006 & 0.338 & 0.209 & 0.163 & $\mathrm{X}$ \\
\hline
\end{tabular}

distribuição dos valores de Kurb bastante aleatória, com uma leve anomalia a noroeste da área estudada, que se aproxima da zona central da cidade. $\mathrm{O} \mathrm{Pb}$ (Figura 1E) apresentou valores de Kurb elevados em vários trechos da área estudada, porém mais significativamente ao norte, nas proximidades do centro histórico da cidade. Nessa região encontra-se instalado um pólo pesqueiro e trata-se da área urbana mais antiga do município, com numerosas edificações do século XIX e meados do século XX. Tanto a manipulação de equipamentos de pesca, como a degradação da pintura dessas edificações já foram descritas como possíveis fontes desse elemento para o material particulado atmosférico. ${ }^{27}$

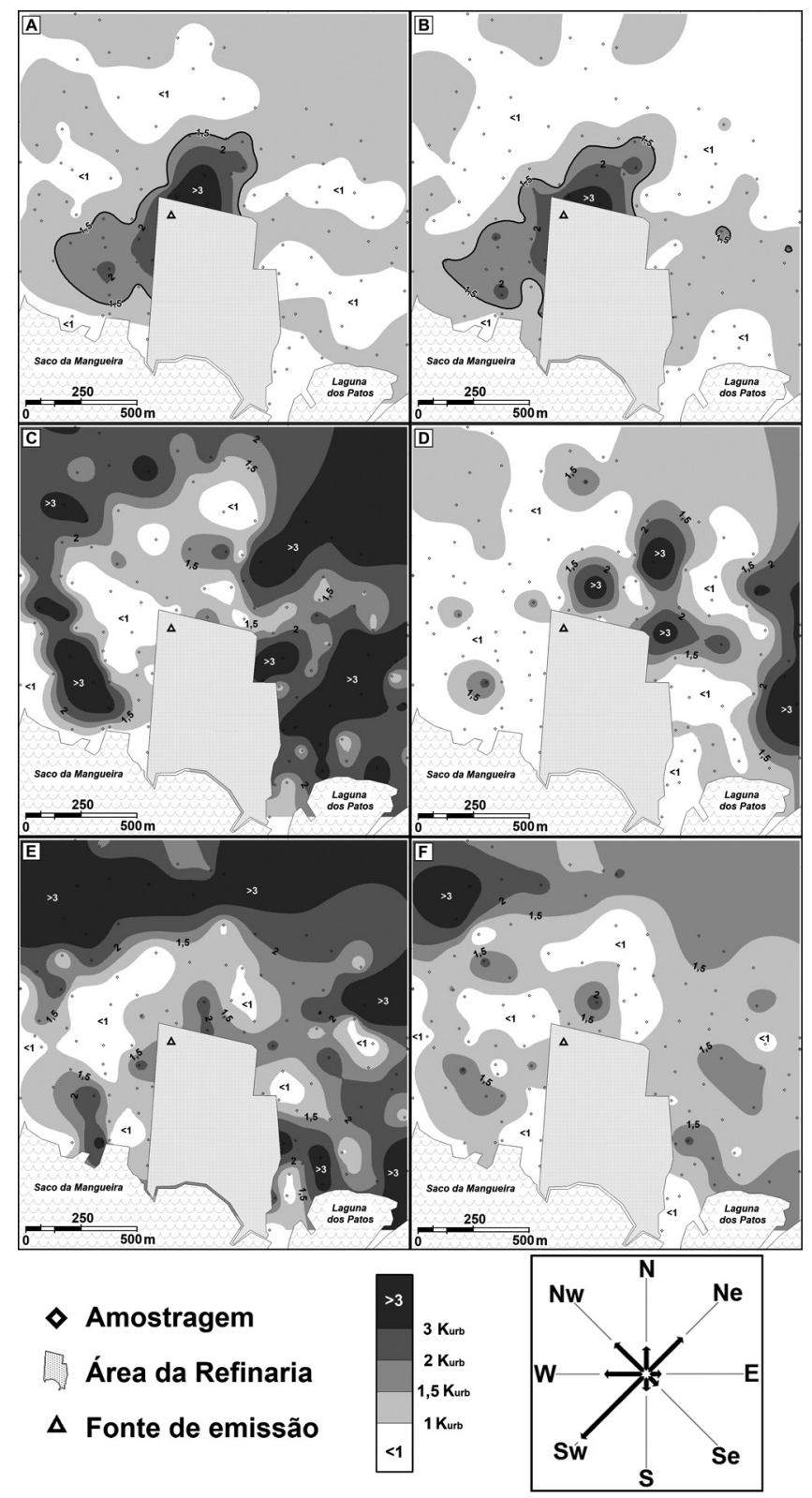

Figura 1. Variação espacial do enriquecimento de $\mathrm{Ni}, \mathrm{V}, \mathrm{Cd}, \mathrm{Cu}, \mathrm{Pb}$ e $\mathrm{Zn}$ (A-F) nos solos superficiais, ao redor da indústria de refino de óleo. A linha preta nos mapas de Ni e V (A e B) correspondem aos limites onde Kurb = 1,5. Os vetores na caixa da legenda representam a frequência de ocorrência do transporte eólico

O Cd apresentou diversas anomalias na área de estudo, com valores de Kurb bastante oscilantes em termos espaciais. No entanto, as anomalias mais significativas encontram-se a leste do domínio 
investigado (Figura 1C), abrangendo as adjacências da zona portuária industrial, onde operava uma indústria de fertilizantes. Admite-se que essa antiga instalação seja responsável pelo enriquecimento de $\mathrm{Cd}$ no local. As indústrias de fertilizantes são reconhecidas por produzirem $\mathrm{Cd}$ para a região estuarina. ${ }^{28} \mathrm{O} \mathrm{Cu}$ apresentou enriquecimento significativo apenas a leste da área estudada (Figura 1D), relacionando-se muito provavelmente com as atividades portuárias.

As distribuições espaciais dos Kurb dos metais Ni e V são similares e são muito diferentes dos demais elementos. Estes metais apresentaram os valores mais elevados exclusivamente próximos às fontes de emissão da refinaria de petróleo. As anomalias de ambos os metais estenderam-se desse sítio em direção sudoeste, de forma extensa e com menor gradiente; já em direção ao norte apresenta extensão mais reduzida e de gradiente mais intenso. Observa-se que as anomalias de Ni e V (Figuras 1A e B), além de restritas às proximidades das fontes de emissão da refinaria, concordam com o padrão de ventos da região. Verifica-se que as velocidades médias dos ventos de qualquer direção são muito semelhantes, portanto, a frequência da direção dos ventos tem um papel fundamental no transporte atmosférico (Figura1). Nesse aspecto se destacam os ventos oriundos de nordeste, seguidos pelos de sudoeste, sudeste e oeste. Esse quadro ambiental é uma confirmação de que a anomalia desses metais é um resultado da dispersão aérea das emissões oriundas da atividade de refino de petróleo. O limiar de Kurb = 1,5 para ambos os elementos aparenta delimitar a abrangência do impacto.

Sendo assim, a população residente no interior da área ocupada pela anomalia tende a ser a mais susceptível ao provável impacto negativo da emissão da refinaria. Em um cálculo aproximativo, as anomalias de Ni e V (limite de Kurb = 1,5) sobrepostas ocupam cerca de $1 \%$ da área urbana residencial do município de Rio Grande, levando a uma estimativa de 1950 habitantes permanentemente impactados pelas emissões. Apesar do cálculo populacional aproximativo, considera-se essa área como uma zona crítica em relação à qualidade ambiental, justificando a implementação de futuros estudos específicos e pontuais sobre a qualidade do ar.

\section{CONCLUSÃO}

Os elementos metálicos Ni e V mostraram ser marcadores muito eficientes do impacto crônico de emissões atmosféricas oriundas de atividades de refino de petróleo. A distribuição dos teores nos solos superficiais demonstra uma forte relação entre a atividade industrial e o padrão de ventos, mesmo sob condições de densa construção urbana. O limiar de 1,5 BGurb para Ni e V demonstra claramente a área de influência das emissões da atividade de refino de petróleo na região urbana de Rio Grande (RS).

Os demais elementos analisados ( $\mathrm{Zn}, \mathrm{Pb}, \mathrm{Cu}$ e $\mathrm{Cd})$ não apresentaram relação com as atividades de refino, possuindo fontes oriundas de outras atividades do complexo portuário urbano-industrial da cidade de Rio Grande.

O estudo realizado apresenta-se como um exemplo da aplicação de marcadores metálicos no solo para mapear a contaminação atmosférica de atividades petroquímicas em uma área urbana, podendo ser reproduzido em outras regiões e servir de diagnóstico indireto da qualidade do ar.

O posicionamento de estações amostrais de qualidade do ar, o estabelecimento de locais para amostragem de compostos aromáticos e a investigação da ocorrência de endemias na população poderão ser adequadamente planejados e implantados, tendo por base a distribuição do Ni e V nos solos.

Sendo assim, o uso dos elementos Ni e V como ferramenta de diagnóstico ambiental mostra-se bastante eficaz, tanto devido aos custos reduzidos, quanto na reprodutibilidade da dispersão das emissões.
A abordagem empregada neste trabalho para o diagnóstico de emissões petroquímicas pode ser reproduzida em outras localidades no Brasil e exterior, servindo de diagnóstico de impacto ambiental e de embasamento para programas de monitoramento de qualidade do ar.

\section{MATERIAL SUPLEMENTAR}

Está disponível em http://quimicanova.sbq.org.br, na forma de arquivo .PDF, com acesso gratuito e compreende a Figura $1 \mathrm{~S}$ e a Tabela $1 \mathrm{~S}$.

\section{AGRADECIMENTOS}

À Agência Nacional do Petróleo (através do PRH-27) e ao CNPq pela concessão de bolsas, assim como à equipe do Laboratório de Oceanografia Geológica da FURG (Tec. E. R. Seus e Acadêmicos G. C. Santos, T. B. Trojan e C. H. S. Campos) pelo apoio nas amostragens e etapas analíticas do estudo.

\section{REFERÊNCIAS}

1. Preuss, R.; Angerer, J.; Drexler, H.; Int. Arch. Occup. Environ. Health 2003, 76, 556, DOI: 10.1007/s00420-003-0458-1; Okona-Mensaha, K. B.; Battershillb, J.; Boobisa, A.; Fielder, R.; Food Chem. Toxicol. 2004, 43, 1103; Lewtas, J.; Mutat. Res./Reviews in Mutation Research 2007, 636, 95, DOI: 10.1016/j.mrrev.2007.08.003.

2. Kodavanti, U. P.; Hauser, R.; Christiani, D. C.; Meng, Z. H.; McGee, J.; Ledbetter, A.; Richards, J.; Costa, D. L.; Toxicol. Sci. 1998, 43, 204; Dye, J. A.; Adler, K. B.; Richards, J. H.; Dreher, K. L.; Am. J. Physiol. Lung Cell Mol. Physiol. 1999, 227, 498; Ghio, A. J.; Silbajoris, R.; Carson, J. L.; Samet, J. M.; Environ. Health Perspect. 2002, 110, 89.

3. Chiffoleau, J.; Chauvaud, L.; Amouroux, D.; Barats, A.; Dufour, A.; Pécheyran, C.; Roux, N.; Aquat. Living Resour. 2004, 17, 273.

4. International Institute for Applied Systems Analysis; The Environmental Impacts of the Gulf War 1991, IIASA: Luxemburgo 2004, IR-04-019.

5. Campen, M. J.; Nolan, J. P.; Schladweiler, M. C. J.; Kodavanti, U. P.; Evansky, P. A.; Costa, D. L.; Watkinson, W. P.; Toxicol. Sci. 2001, 64, 243.

6. De Santis, F.; Fino, A.; Menichelli, S.; Vazzana, C.; Allegrini, I.; Anal. Bioanal. Chem. 2004, 378, 782.

7. Kea, L.; Liua, W.; Wanga, Y.; Russellc, A. G.; Edgertond E. S.; Zheng M.; Sci. Total Environ. 2008, 394, 290.

8. Sadiq, M.; Mian, A. A.; Atmos. Environ. 1994, 28, 2249.

9. Soldi, T.; Riolo, C.; Alberti, G.; Gallorinib, M.; Peloso, G. F.; Sci. Total Environ. 1996, 181, 45.

10. Hanna, S. R.; Britter, R.; Franzese, P.; Atmos. Environ. 2003, 37, 5069, DOI: 10.1016/j.atmosenv.2003.08.014.

11. Venkatram, A.; Isakovb, V.; Yuana J.; Pankratz D.; Atmos. Environ. 2004, 38, 4633, DOI: 10.1016/j.atmosenv.2004.05.018.

12. Harada, Y.; Lee, S.; Babayco, C. B.; Cliff, S. S.; Perry, K. D.; Kelly, P. B.; American Geophysical Union 2005, Fall Meeting , 847.

13. Payet, C.; Bryselbout, C.; Morel, J.; Lichtfouse E.; Naturwissenschaften 1999, 86,484

14. Barwise, A. J. G.; Energy Fuels 1990, 4, 647.

15. Barbante, C.; Boutron, C.; Moreau, A.; Ferrari, C.; Van de Velde, K.; Cozzi, G.; Turetta, C.; Cescon, P.; J. Environ. Monit. 2002, 4, 960, DOI: $10.1039 / \mathrm{b} 208142 \mathrm{c}$

16. FEPAM; LO n $^{\circ}$ 4886/2006DL, 2006.

17. EMS Consultoria; Atlas do desenvolvimento humano no Brasil, 2003.

18. U.S.E.P.A.; Method 3050B (SW-846): Acid Digestion of Sediments, Sludges, and Soils, 1996, Revision 2,12.

19. NRC Canada; Cert. Ref. Mat. data sheets, 2009.

20. Reimanna, C.; Filzmoserb, P.; Garrett, R. G.; Sci. Total Environ. 2005, 
346, 1, DOI: 10.1016/j.scitotenv.2004.11.023.

21. statistXL; Version 1.5; Statistical Power for MS Excel, University of Western Austrália, 2005.

22. Microsoft Professional Edition; Microsoft Officie Excel 2003; Microsoft Corporation, 2003.

23. Golden Software, Inc; Surfer Version 8.00; Surface Mapping System; Golden Colorado, 2002.

24. Beus, A. A.; Grabopvskaia, L. I; Tichonova, N. B.; Environmental Geochemistry, Nedra: Moscow, 1976.
25. Vanz, A.; Dissertação de mestrado, Fundação Universidade do Rio Grande, Brasil, 2000.

26. Saet, Y. E.; Environmental Geochemistry, Nedra: Moscow, 1990.

27. Vanz, A.; Mirlean N.; Baisch P.; Quim. Nova 2003, 26, 25; Mirlean, N.; Robinson, D.; Kawashita, K.; Vignol, M. L.; Conceição, R.; Chemale, F.; Atmos. Environ. 2005, 39, 6204, DOI:10.1016/j.atmosenv.2005.07.002.

28. Baisch, P. R.; Niencheski, F.; Lacerda, L. Em Metals in Coast Environments of Latin America; Seeliger, U.; de Lacerda, L.; Patchinerlam, S. R., eds.; Springer- Verlag: Berlin, 1988. 


\section{MARCADORES METÁLICOS COMO AVALIAÇÃO DO IMPACTO CRÔNICO DE EMISSÕES PETROQUÍMICAS} EM ZONA URBANA

Felipe Azevedo de Paula Garcia*, Nicolai Mirlean e Paulo Roberto Baisch Instituto Oceanográfico, Universidade Federal do Rio Grande, Av. Itália, km 08, s/n, 96201-900 Rio Grande - RS, Brasil

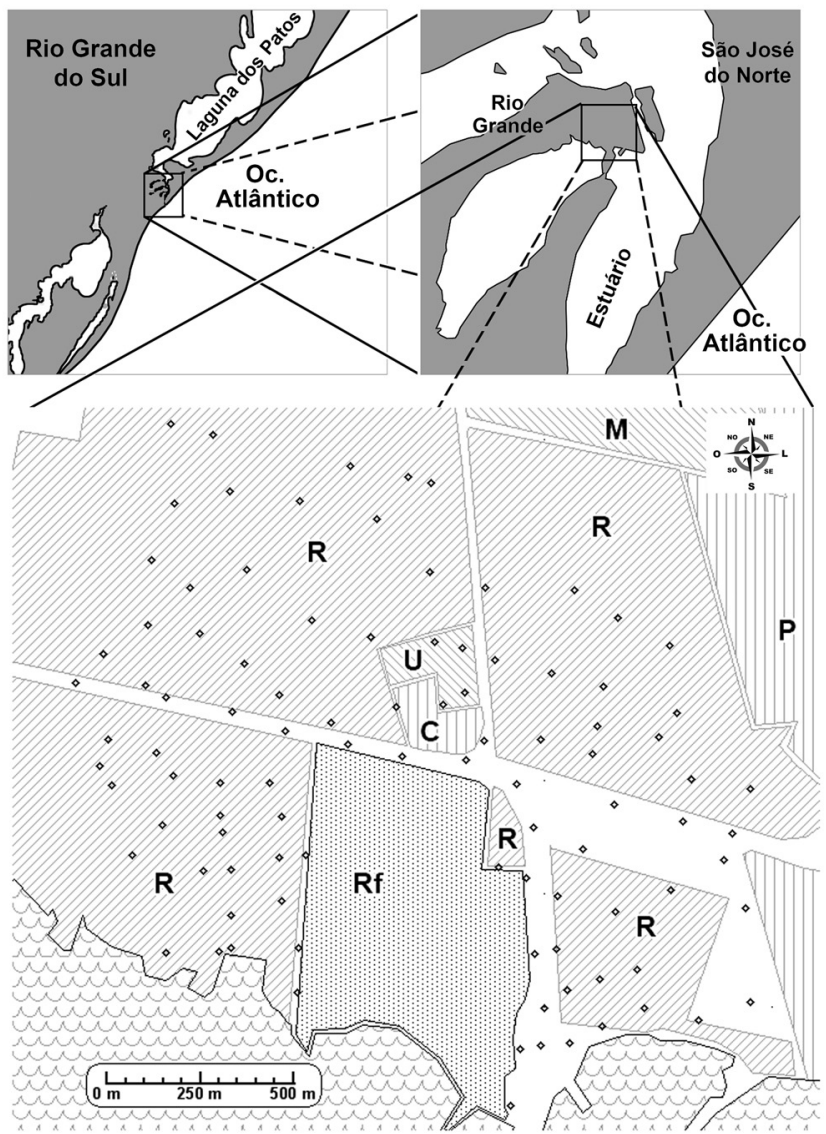

Figura 1S. Localização da área de estudo, distribuição das atividades antrópicas e locais de amostragem de solo. M, Marinha do Brasil; $R$, Residencial; U, Universidade; P, Zona Portuária; C, Companhia de energia elétrica; Rf, Refinaria; $\diamond$, Amostras

Tabela 1S. Ocorrências por classes de teor para cada elemento, com o primeiro pico modal destacado, e estabelecimento do valor de BGurb

\begin{tabular}{|c|c|c|c|c|c|c|c|c|c|c|c|c|c|c|c|c|c|c|}
\hline \multicolumn{3}{|c|}{ Elemento } & \multicolumn{15}{|c|}{ Classes de teor e respectivas ocorrências } & \multirow{2}{*}{$\frac{\mathrm{BG}_{\mathrm{urb}}}{25 \mathrm{mg} / \mathrm{kg}}$} \\
\hline $\mathrm{Ni}$ & $\mathrm{mg} / \mathrm{kg}$ & 5 & 10 & 15 & 20 & 25 & 30 & 35 & 40 & 45 & 50 & 55 & 60 & 65 & 70 & 75 & $>80$ & \\
\hline & ocor. & 0 & 3 & 6 & 16 & 28 & 15 & 13 & 1 & 4 & 6 & 2 & 0 & 1 & 0 & 0 & 3 & \\
\hline \multirow[t]{2}{*}{ V } & $\mathrm{mg} / \mathrm{kg}$ & 10 & 20 & 30 & 40 & 50 & 60 & 70 & 80 & 90 & 100 & 110 & 120 & 130 & 140 & 150 & $>160$ & $40 \mathrm{mg} / \mathrm{kg}$ \\
\hline & ocor. & 0 & 9 & 20 & 23 & 22 & 9 & 5 & 3 & 3 & 1 & 1 & 0 & 0 & 1 & 0 & 1 & \\
\hline \multirow[t]{2}{*}{$\mathrm{Zn}$} & $\mathrm{mg} / \mathrm{kg}$ & 100 & 200 & 300 & 400 & 500 & 600 & 700 & 800 & 900 & 1.000 & 1.100 & 1.200 & 1.300 & 1.400 & 1.500 & $>1.600$ & $400 \mathrm{mg} / \mathrm{kg}$ \\
\hline & ocor. & 3 & 6 & 14 & 22 & 16 & 12 & 13 & 4 & 5 & 1 & 0 & 0 & 0 & 1 & 0 & 1 & \\
\hline \multirow[t]{2}{*}{$\mathrm{Cu}$} & $\mathrm{mg} / \mathrm{kg}$ & 20 & 40 & 60 & 80 & 100 & 120 & 140 & 160 & 180 & 200 & 220 & 240 & 260 & 280 & 300 & $>320$ & $100 \mathrm{mg} / \mathrm{kg}$ \\
\hline & ocor. & 2 & 9 & 12 & 20 & 27 & 2 & 11 & 0 & 3 & 1 & 1 & 2 & 1 & 0 & 0 & 7 & \\
\hline \multirow[t]{2}{*}{$\mathrm{Pb}$} & $\mathrm{mg} / \mathrm{kg}$ & 30 & 60 & 90 & 120 & 150 & 180 & 210 & 240 & 270 & 300 & 330 & 360 & 390 & 420 & 450 & $>480$ & $120 \mathrm{mg} / \mathrm{kg}$ \\
\hline & ocor. & 1 & 7 & 12 & 14 & 12 & 12 & 5 & 5 & 6 & 3 & 5 & 1 & 1 & 1 & 0 & 13 & \\
\hline \multirow[t]{2}{*}{$\mathrm{Cd}$} & $\mathrm{mg} / \mathrm{kg}$ & 0,15 & 0,30 & 0,45 & 0,60 & 0,75 & 0,90 & 1,05 & 1,20 & 1,35 & 1,50 & 1,65 & 1,80 & 1,95 & 2,10 & 2,25 & $>2,40$ & $0,45 \mathrm{mg} / \mathrm{kg}$ \\
\hline & ocor. & 4 & 11 & 21 & 17 & 5 & 7 & 3 & 1 & 8 & 3 & 9 & 2 & 1 & 1 & 0 & 5 & \\
\hline
\end{tabular}

